\title{
AN INTEGRATED APPROACH TO REAL TIME DISPATCHING RULES ANALYSIS AT SEAGATE TECHNOLOGY
}

\author{
Brian Gowling \\ Joel Peterson \\ Seagate Technology \\ 1 Disc Drive \\ Bloomington, MN 55435, USA \\ Madhav Kidambi \\ Applied Materials, Inc. \\ 3425 Hunton Ridge Drive \\ Glen Allen, VA 23059, USA
}

\author{
Tina O’Donnell
}

Seagate Technology

1 Disc Drive

Londonderry, UK

\author{
Daniel Muller \\ Applied Materials, Inc. \\ 71 Longacre Drive \\ Collegeville, PA 19426, USA
}

\begin{abstract}
The challenge of accurately modeling dispatching rules and policies is a difficult and costly exercise. Currently, the only accurate way to qualify the impact and effectiveness of policy changes is by analyzing the policy in either a test facility or in production. In a test facility it is difficult to recreate the production environment. Additionally, to test policy changes in production is risky and can be costly. From a modeling perspective, the duplication of dispatching rules for use in simulation requires time consuming efforts to recreate the dispatching policies as well as the current state of the manufacturing environment. Seagate Technology, working with Applied Materials, has deployed a framework that initializes an AutoSched AP simulation model to the current state of the facility and utilizes the APF Real Time Dispatcher ${ }^{\circledR}$ (RTD) rules in production within the simulation. This paper will present the solution, benefits, and initial results at Seagate Technology.
\end{abstract}

\section{INTRODUCTION}

Challenges often existing in determining the potential impact of continuous improvement initiatives as it relates to mission critical applications. This is especially true when it involves dispatching logic and algorithms used in semiconductor manufacturing due to the volumes and system sensitivities associated with the interrelationship of equipment, buffers, and product mix. Implementation of perceived continuous improvement initiatives can be time consuming and risky as the only way to determine their true effectiveness is through implementation in the production environment itself.

Seagate Technology working with Applied Materials, has implemented a continuous improvement initiatives that addresses the challenges between dispatching and simulation providing the means to understand the impact of dispatching rule changes prior to production implementation.

\section{PROBLEM STATEMENT}

The simulation technology is used for various applications within semiconductor companies. The scenarios includes capacity planning with varying time frames, forecasting, identifying bottleneck tools, validat 
ing dispatching and scheduling policies, and lot release policies. Simulation models are becoming complex as the semiconductor manufacturing scenarios are constantly evolving. The challenges and successes for using simulation can be found in Chien et al.(2008), Fowler et al.(1998), and Scholl et al. (2010) which found that one of the key factors impacting the accuracy of models is the modeling of the local dispatching rules.

There has been significant research performed in evaluating dispatching rules and scheduling polices within the simulation environment. The studies include the evaluation of commonly used dispatching rules against the mathematical programming based algorithms. These studies focus on the impact of the dispatching and scheduling policies against the cycle time and throughput for bottleneck tools in the factory. Some examples can be found in Sunkara et al. (2011), Hsieh et al. (2007), Rose (1999), Khul et al (2004). One of the papers written, Bureau et al. (2007), lists that one of limitations in simulating the consistent global and local scheduling is that dispatching rules used in the simulation model are far from reality.

Seagate Technology utilized dispatching software that takes into account the current state of the factory in determining where to best route product. Decision rules used in the software can be very complex in an effort to operate the facility as efficiently as possible. Due to the nature of the application it can be difficult to foresee the effects of rule changes as the conditions in the factory change due to downtime, product mix, etc.

\subsection{Current Dispatching Approach}

Seagate utilized Applied Material's APF Real Time Dispatcher (RTD) product, or "dispatcher" henceforth, in its manufacturing environment to assist in controlling the flow of material and in the real-time decision making processes. Figure 1 depicts an example of this dispatcher configuration.

Seagate also uses AutoSched AP® (ASAP) for the factory online simulation model, or "online simulation". The online simulation of dispatch rules is very basic at the present. The ASAP software also provides a customize extension to allow for complex rule development beyond the standard library of rules. The extension capabilities cannot duplicate the dispatch rules in the same manner that rules are deployed within the dispatcher environment.

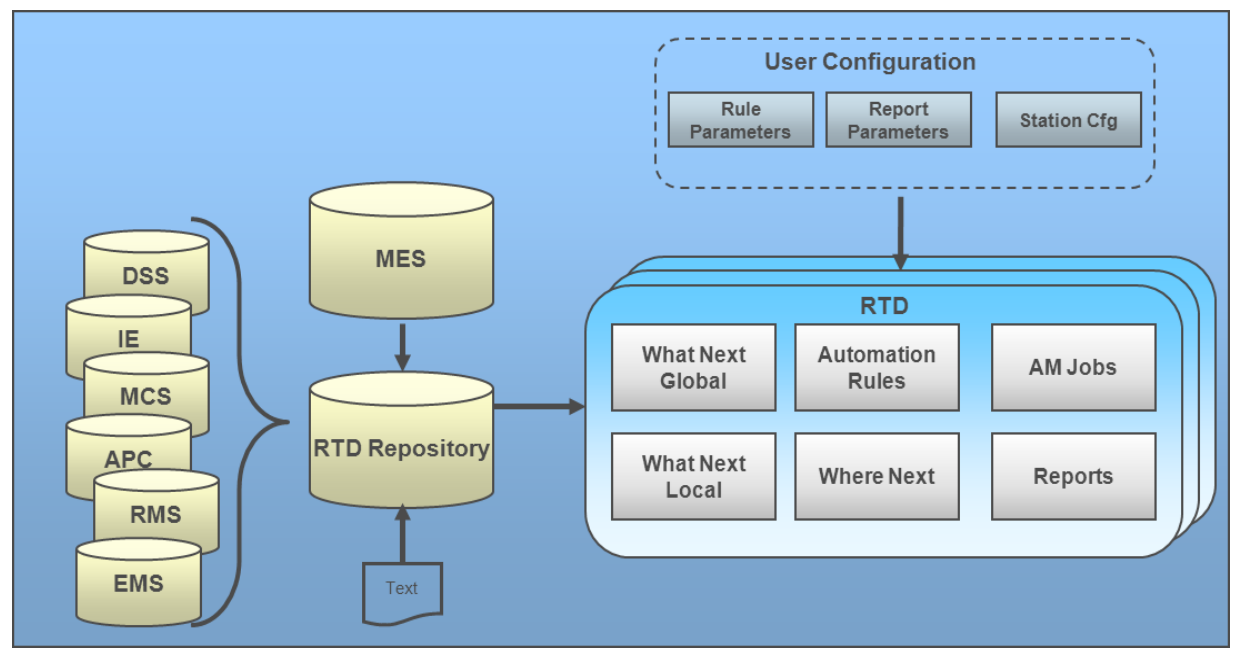

Figure 1: Sample dispatcher configuration.

The current dispatching method used within the Seagate online simulation model is as follows:

- Lots are grouped by recipe 


\section{Gowling, Peterson, O’Donnell, Kidambi and Muller}

- Batches are ranked by Priority and First-in, First-out (FIFO)

- Batches have minimums and maximums quantities based on equipment and process step.

The dispatcher rule methodology used within the factory is:

- Lots are grouped by recipe

- Batches are ranked by Priority (manipulated via user parameter table), FIFO, and several other parameters

- Management of dispatcher rules is done via a user parameter table applied to indexed by Tool, Product, Process Step, Process Path, Lot Type, Route Type, RD Team, Experiment, Recipe, Priority and Lot Name. Between 5,000 and 10,000 parameters are active on any given day.

- Over 40 parameters control not only how lots are prioritized, routed, and manipulated, but also how tool resources are utilized and qualified.

- In additional to static parameter settings, several rules are dynamically enabled based on a variety of WIP and tool conditions.

- Each parameter within the dispatcher rules is effectively a custom extension.

Within the dispatcher, decision rules are developed that take into account the current state of the FAB in determining the routing of products to help maximize system performance. Rules often take into account large amounts of data from various sources and are often very complex in an effort to make the best decisions at that instant.

\subsection{Challenges}

Challenges existed within Seagate's current environment that have provided Seagate and Applied Materials with the opportunity to address the analytical capabilities through the integration of the two products. Some of the challenges that Seagate posed to Applied are as follows:

- How can Seagate users experiment and validate the dispatching \& scheduling policies through tradeoffs of conflicting business goals without the risk of trial and error in a production environment? Dispatching rule changes can adversely affect a factory's key outputs such as; product cycle time, equipment capacity, utilization and throughput.

- How can Seagate demonstrate that a dispatching algorithm change needed for continuous improvement plans will not adversely affect production schedule and/or R\&D goals.

- How can Seagate users record factory events and determine a more efficient response to unforeseen events such as tool down, WIP bubbles, demand changes, etc.?

- How can Seagate address the online simulation forecast accuracy gaps between basic simulation dispatch rules and complex rules in the dispatcher?

\subsection{Current Simulation Approach}

The current online simulation at Seagate uses a typical approach to modeling as seen in Scholl (2011). It is used to forecast up to 7 days and is used in a variety of applications like bottleneck detection, throughput goals, and forecasting planned down events. As discussed previously in section 2.1, the simulation uses basic dispatching rules. This results in many of the gaps between the production and simulated factory when attempting to accurately forecast any particular lot's behavior over a period of time. Figure 2 summarizes the typical defects observed in the online simulation at a lot-level basis. In this case, a defect represents the delta between an actual lot's step count and a simulated lot's step count, and that delta is greater than an allowable threshold over a period of time. 


\section{Forecast Accuracy: Avg. Count of Lot-by-lot defects > Threshold}

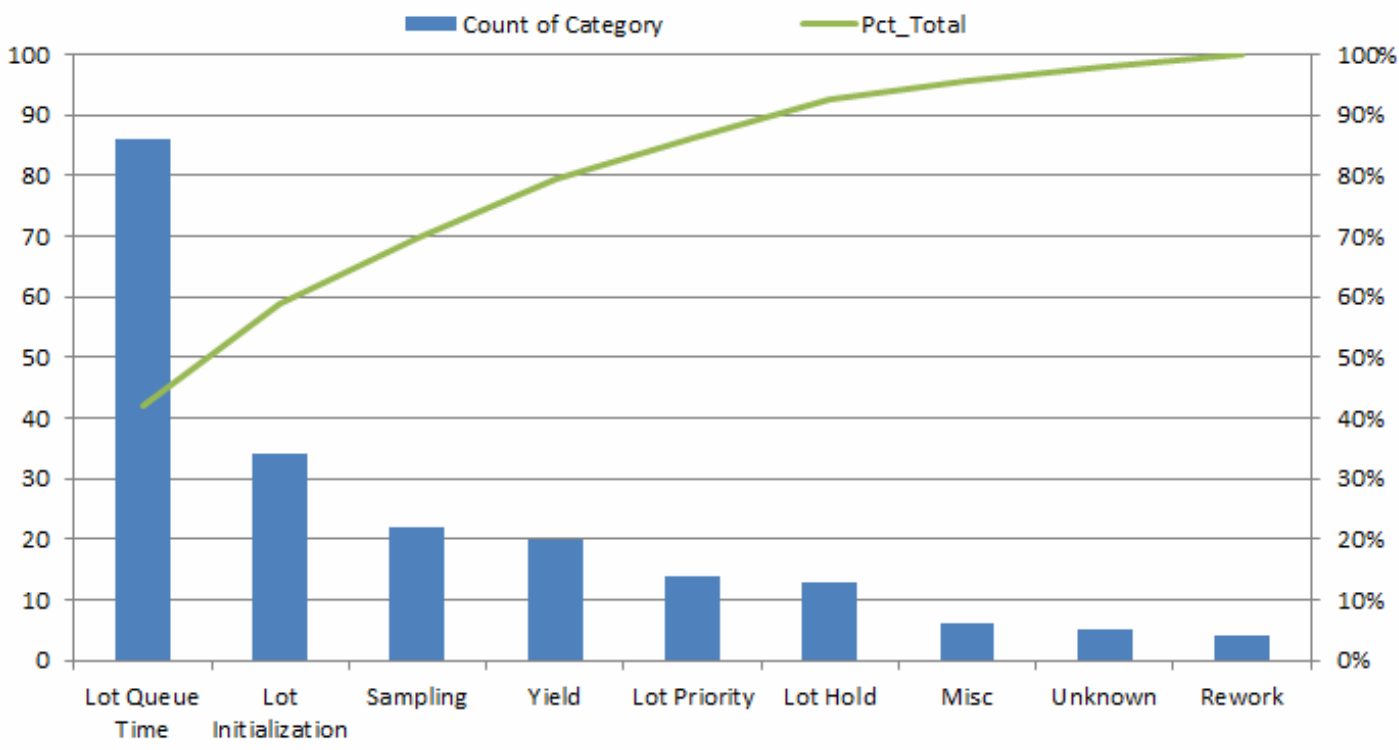

Figure 2: Lot-by-lot defect comparison of simulation vs. production.

Automating dispatch rule conversion in the online simulation is expected to have a significant impact on the current online simulation's lot-level accuracy. The opportunity for improvement is largely due to the fact that greater than about $40 \%$ of the lot-by-lot simulation deltas are caused by major differences in a lots' queue times. This, in-part, is due to the lack of modeling specific dispatch rules. Essentially, in the current simulation lots are processed in a different order than they would in production and arrive at downstream operations in a different sequence. During the course of this project Seagate will be measuring the improvements in lot queue times defects.

Lastly, it should be noted that Seagate's online simulation has been relatively accurate at a factory-level on average, but is less accurate for individual lot-level forecasting. As the demand for applications of the online simulation increase, so does the need for improvements in simulation forecast accuracy.

\section{DISPATCHING AND SIMULATION}

\subsection{Past and Present Approaches}

There have been numerous effort to model dispatching rules in a simulation environment. Here are some past and current approaches.

\subsubsection{Standard Simulation Rules}

The current online simulation product includes a very large library of rules. A simulation user can attempt to utilize these rules to best closely matching that of the dispatching system. These standard rules tend to focus on individual equipment and lot selection rules to help maximize equipment performance. The rules tend to be limited in their complexity to utilize system wide data to evaluate the condition necessary to make near optimal dispatching decisions to help maximize system performance. 


\subsubsection{Rule Compiler}

In the past a rule compiler existed that compiled dispatcher rules to be directly used in the simulation environment. Over time this effort was discontinued do to the following limitations:

- Testing indicated rules were too slow for simulation

- Projected cost to maintain was expected to be high

- Components of dispatcher capabilities were not supported (SQL blocks, data blocks, procedure blocks)

\subsubsection{C++ Extensions}

The current approach of model developers desiring to model custom rules from the dispatcher to the online simulation is through $\mathrm{C}++$ extension development. Figure 3 depicts this approach. Customers duplicate the dispatching rules as close as possible in extensions that get compiled within the simulation environment. Some of the benefits and limitation of this approach are as follows:

- The rules tend to perform well with respect to the desired speed of simulation.

- Cost to maintain the rules are high as $\mathrm{C}++$ extension development is required.

- Rules are duplicated and assumptions must be made to accommodate the differences between products.

- Dispatching users and simulation users may be in different departments and have limited knowledge of each other's product.

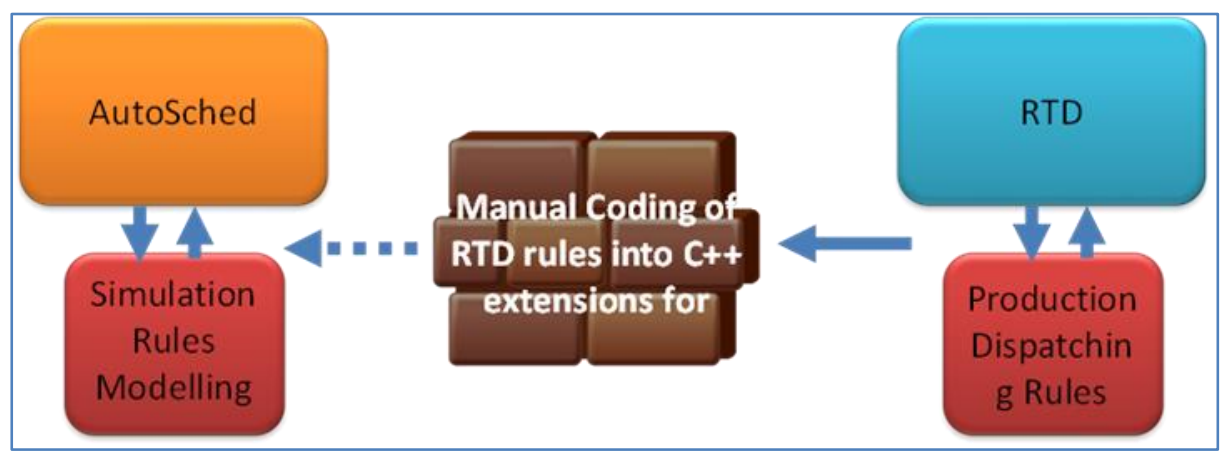

Figure 3: Current approach for modeling the dispatch rules in the factory online simulation.

\subsection{Design Approach}

In analyzing the dispatcher rule requirements and simulation environment necessary to implement rules directly the following was discovered:

- In most rules, $2 / 3$ of the time or more is used in preparing the data (i.e., joins, field manipulation, data consolidation, etc.) for dispatching and $1 / 3$ of the time is in rule execution. Figure 4 depicts the major dispatcher rule functional breakdown based on analysis of customer rules.

- In the online simulation rules, most of the data preparation is done in loading of the simulation instead of at execution time. 
Gowling, Peterson, O’Donnell, Kidambi and Muller

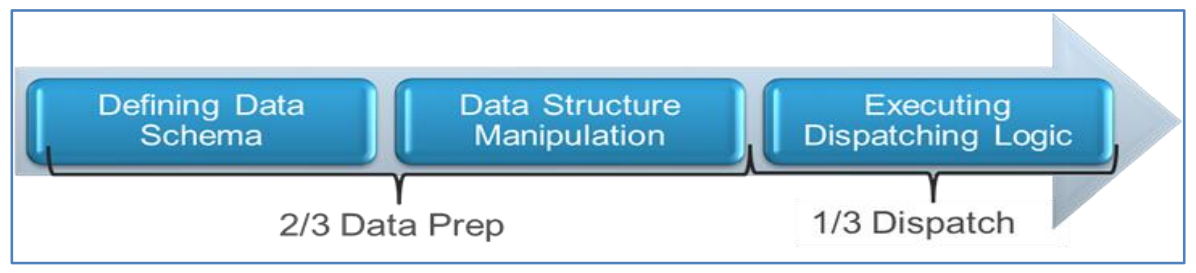

Figure 4: Analysis of customer's dispatcher rule functions.

Through this analysis it was determined that $2 / 3$ of the rules, along with the rule processing, could possibly be ignored if the simulation model could execute the remaining $1 / 3$ dispatching logic. This provided the motivation for a possible solution overcoming the performance barriers of the past.

One of the major benefits of simulation is the accelerated speed proving users with the results in a matter of minutes or even seconds, the desire to test production rules in a compressed time could provide users with huge benefits. As previous efforts in direct rule use have been hampered due to performance, it was determined through customer input that an acceptable 1-week simulation run would need to be completed in no more than 2 hours. Table 1 highlights the simulation rule execution calculation needed for market acceptance as deemed by users.

Data utilizing actual dispatcher rule execution times was used to help test the execution requirements within a simulation model. Based on prototyping utilizing customer's rule in and integrated the dispatcher and online simulation environment, promising results indicated a potential for $233 \mathrm{x}$ rule execution improvement in the online simulation environment as summarized in the table below.

Table 1: Analysis of rule execution requirements within a simulation model.

\begin{tabular}{|l|l|l|}
\hline \multicolumn{3}{|c|}{ Dispatching Rule Simulation Goal is to Simulate 1 week in 2 hours } \\
\hline Average Dispatcher Execution Time & 4 & seconds / dispatch \\
\hline Simulation Window & 1 & simulated week \\
\hline Simulation Execution Time & 2 & real hours \\
\hline Estimated Daily Dispatch Rate & 60,000 & dispatch requests / day \\
\hline Dispatch Requests in Simulation over Window & 420,000 & dispatch requests / week \\
\hline Simulation Dispatch Speed & 0.017142857 & real seconds / dispatch \\
\hline Required Improvement Factor & $\underline{\mathbf{2 3 3 X}}$ & real speed/sim speed \\
\hline
\end{tabular}

Through this effort the performance barriers of utilizing dispatch rules directly within an online simulation provided the potential for users to expand their use of simulation in their dispatching operations.

\subsection{Perceived Benefits}

Through the integration of the dispatching rules into the simulation environment the following benefits can be recognized dependent upon individual use cases.

\subsubsection{Integrated/Shared Data Modeling Capabilities Across Products}

The integration of the dispatching and simulation products simplifies the process of initializing the simulation model to the current state of the manufacturing facility. The current state of equipment, WIP, as well as order release and preventive maintenance schedules can be included as this data now can be shared across the software platforms. Below are shared data elements and benefits. 
- Factory system data availability throughout the reporting platform.

- Reduced online simulation model development time and updating efforts

- Similar reporting formats for both the dispatcher and online simulation.

\subsubsection{Expanded Analytical Capabilities}

The major benefits of the integrated dispatching and simulation solution is the potential to proactively work within the production system environment with actual data as part of a continuous improvement initiatives. Some of the expected capabilities of the integration are:

- Evaluate dispatch rule changes against future impact (orders/equipment/etc.)

- Test dispatch rule performance against historical performance (existing data)

- Determine impact of unscheduled down events

- Preempt potential down events through "smart" tool analysis

- Determine equipment deployment plans

- Look-ahead/predictive modeling capability

- Capacity planning capability

\subsubsection{Reduced Total Cost of Ownership}

The expected benefits with the shared data model and analytical capabilities greatly contributes to lowering the Total Cost of Ownership (TCO) for users. Savings and improvements can be recognized in the following areas:

- Dispatcher rules deployment

- Offline testing and evaluation reduced risk and increases confidence

- Reduced training costs as product integration simplifies model development and maintenance

- Reduces simulation modeling costs

- No need to duplicate dispatching rules with $\mathrm{C}++$ rules

- Automatic model initialization of current FAB state with integrated data

- Common reports between the dispatcher and the online simulation

\section{IMPLEMENTATION PLAN}

The current Seagate implementation plan consist of a 4-phased approach. The following paragraphs will describe the planned phased approach.

\subsection{Phase I}

In this phase Applied developed and delivered the capability to integrate the default rule in from the dispatcher into an online simulation. Key validation and debugging functionalities are were also developed. The key debugging features allows Seagate to validate the dispatcher rule behavior in the simulation. Key rule statistics are collected to monitor the dispatcher rule performance. The online factory simulation model can be paused at a certain point of time to analyze the behavior of dispatcher rules. All the data at the end of the simulation can be captured in a virtual repository which later can be used for doing validation and what-if analyses.

Also in this phase, a custom lot-level event logger was integrated into the simulation to capture lot events for each process step the lot experienced. This assists in the validation of rule execution as well as 
providing tracking information to determine the simulation accuracy improvements. The information the event logger captures includes:

- Lot ID and priority

- Timestamps for arrival at the step including: reserving a tool, track in/out, and process start/stop for calculation of processing and non-processing cycle time classifications.

- Tool ID the lot was processed on

- Route step name and number

\subsection{Phase II}

The Phase II focus is to develop a mechanism to periodically refresh the input files used in the simulation. This capability will allow the dispatch rules to use historical information during the simulation run time. In addition to this capability, improvements will be made to the logging \& validation methods.

\subsection{Phase III}

In Phase III, the focus is to enhance and deploy the capabilities developed in Phase II. i.e use the historical information from the online simulation model in the dispatch rule.

\subsection{Phase IV}

Phase IV is to focus on the conditional logic in dispatch rules. This will ensure that the same rule can be used for both production dispatching and the simulation model.

\section{INITIAL RESULTS}

After Phase I, many results have already been achieved. The project has already validated that five of the forty unique dispatch parameters have been successfully completed during the first week implementation. Previously developing five custom rules would likely have considerable longer to code and test in $\mathrm{C}++$.

Although there are several steps required to verify that dispatch rules are being modeled accurately, Figure 5 below summarizes one of these steps. This figure displays a side-by-side comparison of a single dispatch rule's parameter with changes over a simulated period and its effect on average WIP (FWLAVG) and average batch size (BATCHSZAVG). The rule that is verified is LARGEST_BATCH_FIRST which is a user defined WIP limit at which a tool's local dispatching policy changes to prioritizing the largest batch first over all other policies (with a few minor exceptions) until WIP is reduced to less than the WIP threshold parameter. 


\begin{tabular}{|c|c|c|c|c|c|c|c|}
\hline & \multicolumn{2}{|c|}{ Basic Rules } & \multicolumn{4}{|c|}{ Automatic RTD Rule Conversion (Default Rules) } \\
\hline & & \multicolumn{2}{|c|}{ Baseline Sim } & \multicolumn{2}{|c|}{$\begin{array}{c}\begin{array}{c}\text { LARGEST_BATCH_FIRST } \\
=75\end{array} \\
=75\end{array}$} & \multicolumn{2}{|c|}{$\begin{array}{c}\begin{array}{c}\text { LARGEST_BATCH_FIRST } \\
=1\end{array} \\
\end{array}$} \\
\hline PERIOD & STNFAM & FWLAVG & BATCHSZAVG & FWLAVG & BATCHSZAVG & FWLAVG & BATCHSZAVG \\
\hline Period-01-Day & TOOL-A & \begin{tabular}{|r|}
4.53 \\
\end{tabular} & \begin{tabular}{r|}
1.72 \\
\end{tabular} & 1.03 & 1.75 & \begin{tabular}{|l|}
0.63 \\
\end{tabular} & 1.76 \\
\hline Period-01-Night & TOOL-A & 6.2 & 1.81 & 5.41 & 1.76 & 6.62 & 2.04 \\
\hline Period-02-Day & TOOL-A & 7.09 & 1.78 & 2.85 & 1.46 & 2.81 & 1.5 \\
\hline Period-02-Night & TOOL-A & 3.02 & 1.86 & 1.48 & 1.7 & 1.61 & 1.75 \\
\hline Period-03-Day & TOOL-A & 3.47 & 1.6 & 2.49 & 1.83 & 2.08 & 1.81 \\
\hline Period-03-Night & TOOL-A & 3.16 & 1.65 & 6.38 & 1.92 & 6.91 & 2.1 \\
\hline Period-04-Day & TOOL-A & 54.61 & 2.94 & 33.15 & 2.88 & 47.22 & 6.4 \\
\hline Period-04-Night & TOOL-A & 101.34 & 4.38 & 73.9 & 3.51 & 73.52 & 4.47 \\
\hline Period-05-Day & TOOL-A & 146.54 & 3.86 & 123.06 & 10.53 & 99.3 & 5.67 \\
\hline Period-05-Night & TOOL-A & 155.9 & 4.05 & 126.69 & 5.46 & 80.32 & 4.76 \\
\hline Period-06-Day & TOOL-A & 172.85 & 3.85 & 153.69 & 9.41 & 104.07 & 6.91 \\
\hline Period-06-Night & TOOL-A & 187.84 & 3.12 & 123.89 & 3.82 & 101.17 & 3.35 \\
\hline Period-07-Day & TOOL-A & 169.79 & 3.89 & 78.35 & 2.47 & 34.65 & 1.81 \\
\hline Period-07-Night & TOOL-A & 142.11 & 5.3 & 22.75 & 1.55 & 3.36 & 1.6 \\
\hline
\end{tabular}

Figure 5: Verification of a single rule change.

In the baseline simulation, where LARGEST_BATCH_FIRST is not present, an increase in WIP is introduced on Period-04-Day and continues through the rest of the remaining 7-day simulation increasing the batch size moderately duration that time. In the second scenario, LARGEST_BATCH_FIRST is read in using the automated dispatch rule conversion in the online simulation and the parameter is set to 75 . As the simulation progresses and the WIP approaches this limit the batch size drastically jumps to 10.53 from 3.51 the period before. The tool continues to have a higher batch size over the next few periods until the large batches are less common and the WIP is worked off, which also happens much sooner than the baseline simulation. Lastly, in the third scenario, the LARGEST_BATCH_FIRST is set to 1 which effectively turns the rule on for any dispatch batch. Notice that as the WIP event appears on Period-04-Day the batch size is $\sim 2 \mathrm{x}$ higher than in the previous two scenarios. This type of analysis could allow for an optimal parameter setting of this particular rule depending on factory conditions.

\section{NEXT STEPS}

As of June 2013, Phase 2 of the Implementation Plan is being executed. Applied Materials will be focusing on developing the capabilities as outlined in the Phase 3 and 4 of the Implementation Plan to improve capabilities to replicate the production dispatching rules in the simulation model and to further reduce the cost of ownership to develop and maintain dispatching rules within the simulation model.

Seagate will continue to work closely with Applied Materials while starting to incorporate the capabilities developed in making decisions for validating dispatching rules and other planning scenarios.

\section{ACKNOWLEDGMENTS}

The authors of this paper would like to recognize the following individual for their contribution and support to the project:

Seagate Technology

Joe Niemi

Kevin Monson

Giles Doherty

Richard Surman
Sr. Manager IT Manufacturing Systems

Sr. Staff Analyst IT Manufacturing Systems

Staff Industrial Engineer

Staff IT Engineer 
Gowling, Peterson, O’Donnell, Kidambi and Muller

Applied Materials

Paul Hoad

APF Technologist

Atsushi Isobe

ASAP Technical Support

\section{REFERENCES}

Bureau, M., S. Dauzere-Pérès, C. Yugma, L. Vermariën. 2007. “An Approach for Simulating Consistent Global and Local Scheduling." IEEE/SEMI Advanced Semiconductor Manufacturing Conference. 96-99.

Fowler, J. M. Fu, L.W. Schruben, S. Brown, F. Chance, S. Cunningham, C. Hilton, M. Janakiram, R. Stafford, J. Hutchby. "Operational Modeling \& Simulation in Semicondutor Manufacturing." Proceedings of the 1998 Winter Simulation Conference, edited by D.J. Medeiros, E.F. Watson, J.S. Carson and M.S. Manivannan. 1035-1040.

Hsieh, B., C. Chen, S. Chang. 2007. "Efficient Simulation-Based Composition of Scheduling Policies by Integrating Ordinal Optimization With Design of Experiments." IEEE Transactions on Automation Science and Engineering 4 (4): 553-568.

Kuhl, M., G. Laubisch. "A Simulation Study of Dispatching Rules and Rework Strategies in Semiconductor Manufacturing." 2004 IEEE/SEMI Advanced Semiconductor Manufacturing Conference. 325329.

Rose, O. "Estimation of the Cycle Time Distribution of a Wafer Fab by a Simple Simulation Model." Proceedings of the SMOMS '99 (1999 WMC), page 133-138.

Scholl, W., B.P. Gan, D. Noack, P. Preuss, P. Lendermann, O. Rose, F.S. Pappert. "Implementation of a Simulation-Based Short-Term Lot Arrival Forecast in a Mature 200mm Semiconductor Fab." Proceedings of the 2011 Winter Simulation Conference, edited by S. Jain, R.R. Creasey, J. Himmelspach, K.P. White, and M. Fu. 1932-1943.

Scholl, W., B.P. Gan, D. Noack, P. Preuss, M.L. Peh, P. Lendermann, and O. Rose. "Towards Realization of a High-Fidelity Simulation Model for Short-Term Horizon Forecasting in Wafer Fabrication Facilities." Proceedings of the 2010 Winter Simulation Conference, edited by B. Johansson, S. Jain, J. Montoya-Torres, J. Hugan, and E. Yücesan, 2563-2574.

Sunkara, R., R. Rao. "Use of Discrete Event Simulation to Analyze Dispatch Policies of an Equipment Group in Semiconductor Fab." Proceedings of the 2011 Winter Simulation Conference, edited by S. Chick, P.J. Sánchez, D. Ferrin, and D.J. Morrice. 1474-1479.

\section{AUTHOR BIOGRAPHIES}

BRIAN GOWLING works as a Senior Industrial Engineer of modeling and simulation for Seagate Technology at the wafer fab in Bloomington, MN. He received a B.S in Industrial Engineering in 2002 and an M.S. in 2003 from The University of Wisconsin. He joined Seagate in 2007 and is responsible for the fab simulation. His email address is brian.j.gowling@seagate.com.

JOEL PETERSON works as a Staff Program/Project Manager within Factory Control Systems Group at the wafer fab in Bloomington, MN. He received a B.A. in Elementary Education in 1991 from Concordia College. He started with the Production Control group at Seagate in 1995 and moved to the MES group as a 6 Sigma Black Belt in 2000. He is responsible for creating and deploying RTD rules. His email address is joel.n.peterson@seagate.com.

TINA O'DONNELL works as a Senior MES Engineer for Seagate Technology at the wafer fab in Springtown, Derry, Northern Ireland. She received a BEng in Electronic Engineering and Computing in 2002 and a PhD in Optimization of the Bullwhip Effect in Supply Chains in 2007 from University of Ul- 
ster. She is responsible for creating and deploying RTD rules. Her email address is tina.o'donnell@seagate.com.

MADHAV KIDAMBI has been working in the semiconductor industry for the past 14 years in the areas of Simulation modeling, Dispatching /Scheduling and Factory Automation Systems . Mr. Kidambi has led the deployment of the APF RTD \& Reporter software in $200 \mathrm{~mm} \& 300 \mathrm{~mm}$ Front end manufacturing sites. He has also worked in the scheduling and simulation with the AutoSched AP \& SmartSched products from Applied Materials. Prior to joining Applied Materials, he has worked at Infineon, Qimonda and Spansion. He is currently the Product Manager for APF/RTD and Activity Manager software from Applied Materials. Madhav Kidambi has a MS in Industrial \& Systems Engineering from Virginia Tech University and BE in Mechanical Engineering from VNIT, India. His e-mail address is madhav_kidambi@amat.com.

DANIEL MULLER has worked for Applied Materials since 1992. He is currently the Product Manager for the AutoMod and AutoSched Simulation Products. He previously had worked as a Consulting Manager, World-Wide Presales, and as a Senior Simulation Analyst, conducting numerous simulation studies for over the ten years in a variety of industries. He currently coordinates the simulation development activities as well as manages the AutoMod world-wide distributor network. Mr. Muller received a B.S in Industrial Engineering from Georgia Tech in 1985 and an M.S. in Industrial Engineering from The University of Pittsburgh in 1991. He is a senior member of IIE and has served as Business Chair for the '01 and'04 Winter Simulation Conferences. His email address is dan_muller@amat.com. 